\title{
The Influence of Psychological Stress on HPV Infection Manifestations and Carcinogenesis
}

\author{
Liborija Lugović-Mihića,b Hrvoje Cvitanovićc Ivka Djakovićd \\ Matea Kuna ${ }^{a, b}$ Ana Šešerko ${ }^{e}$ \\ aDepartment of Dermatovenereology, University Hospital Center Sestre Milosrdnice, Zagreb, \\ Croatia, 'bSchool of Dental Medicine, University of Zagreb, Zagreb, Croatia, 'Department of \\ Dermatovenereology, Karlovac General Hospital, Karlovac, Croatia, dDepartment of of Gynaecology and \\ Obstetrics, University Hospital Center Sestre Milosrdnice, Zagreb, Croatia, 'Department of Gynaecology \\ and Obstetrics, University Hospital Center Zagreb, Zagreb, Croatia
}

\section{Key Words}

Psychological stress - HPV infection - Carcinogenesis - Immune response • Psychoneuroimmunology

\begin{abstract}
Psychological stress is an important factor involved in disease manifestations of human papillomavirus (HPV) infection, and it can participate in HPV-associated carcinogenesis. The impact or effect which stress can have (exert) depends on a person's genetic pool, experiences and behaviors. Due to inconsistencies in some study results, this issue remains a subject of research. Concerning the course of HPV manifestations, it has been observed that a higher number of life stressors in at least the previous 6 months, the absence of social support and the types of personal coping mechanisms employed, all influence HPV progression. In women with cervical dysplasia, a connection between greater stress experiences and dysregulation of specific immune responses has been observed. Once HPV enters a cell via the $\alpha 6$ integrin there are three possible sequences: latent infection, subclinical infection, and clinically manifest disease. HPV proliferation in differentiated epithelial cells induces morphologically cytopathic changes (koilocytosis, epidermal thickening, hyperplasia, hyperkeratosis). Oncogenic transformation requires the integration of the virus genome into the host genome. In doing so, DNA in the E1 region of E2 breaks down, leading to transcription disorders of E6 and E7. For the formation of irreversible malignancy, the following sequence is necessary: initial expression of E6 and E7 genes followed by suppression of apoptosis and the stabile expression of E6 and E7 proteins that protect transformed cells from apoptosis. A successful immune response is characterized by a strong, local cell-mediated immune response. Several factors are important for the regression of HPV manifestation/infection, among which is psychological stress which can prolong the duration and severity of HPV disease. Stress hormones may reactivate latent


tumor viruses, stimulate viral oncogene expression, and inhibit antiviral host responses. In the regression of HPV infection, increased activity of Th1 cells was observed. However, during psychosocial stress, a decrease in the Th1 type of immune response is seen, and there is a shift towards a Th2 response. Understanding perceived stress and biological changes in stress, as well as the evaluation of immune parameters, gives researchers a better picture of how stress influences HPV infections and how to improve disease management and outcomes.

(C) 2021 The Author(s). Published by Cell Physiol Biochem Press GmbH\&Co. KG

\section{Introduction}

Psychological stress is an important factor involved in disease manifestations of HPV infection, and it can participate in carcinogenesis associated with HPV. Due to inconsistencies in some study results, this issue remains a subject of research. It is important to determine to what extent stress plays a role in HPV manifestations and carcinogenesis and how much it participates in the onset, development, and progression of infections.

\section{Features of HPV}

Human papillomavirus (HPV) is a DNA virus that belongs to the Papillomaviridae family. It is one of the most ubiquitous viral infections in humans, and it usually manifests as skin or genital mucosa lesions, although it can occur in other mucosa as well. It has long been known that most sexually active people will be infected by this virus at some point of their life, and the incidence of HPV infection is highest in the age group for those 20-40 years old [1-4]. Despite its high prevalence, most who get infected will not have a clinically overt infection. Still, the persistence of the HPV infection may cause a higher risk for developing cervical intraepithelial neoplasia (CIN) and invasive carcinoma. In women, a breakout from a highrisk HPV infection typically takes 14 months to clear up for an oncogenic infection and 5-6 months for a non-oncogenic infection [4].

To date, more than 200 types of HPV have been identified [5]. A strain is considered new when the nucleotides of the L1 part of the HPV genome differ from that of known HPV viruses by more than $10 \%[6,7]$. HPV viruses can be divided into different groups by their affinity for certain tissues, which in some part depends on their genotype [6]. The specific skin lesions for each type of HPV are as follows: common warts - types 2, 7, 22; plantar warts - types 1, 2, 4, 63; flat warts - types 3, 10, 28; and for verrucous cyst type 60 and epidermodysplasia verruciformis - more than 15 different types. There are also specific HPV types for anal/genital manifestations: anogenital warts-types 6, 11, 42, 44 and others; anal dysplasia (lesions) - types 16, 18, 31, 53, 58; and genital cancers- highest risk types 16, 18, 31,45 , other high-risk types - 33, 35, 39, 51, 52, 56, 58, 59, and probably high-risk- types $26,53,66,68,73,82$ [5]. The types of HPV for oral/oropharingeal lesions are: focal epithelial hyperplasia (mouth) - types 13 and 32; mouth papillomas-types 6, 7, 11, 16, 32); and oropharyngeal cancer-type 16; laryngeal papillomatosis-types 6 and 11 [5]. Concerning oncogenic risk, the low-risk HPV types are $6,11,49,42,43,44,54,61,70,72$ and 81, while the high-risk types are $16,18,31,33,35,39,45,51,52,56,58,59,68,73$, and 82 . In addition, $60 \%$ of all genital warts are caused by HPV types 16 and 11 [8].

HPV is transmitted through direct skin or mucous membrane contact, and infection can be clinical, subclinical or latent. Microimpairments in the skin or mucous membrane enhance transmission, but for infection to develop it is essential for the complete virus to be transmitted, not only its DNA fragments [4]. In the sexually active population, HPV is present in $80 \%$ of women of reproductive age, but its manifestations resolve-spontaneously in most cases (cca $80 \%$ of infections) within 12-24 months [9]. Untreated HPV manifestations in men can cause invasive penile carcinoma, which represents $1 \%$ of malignancies in men in developed countries and 10-20\% in undeveloped countries. In men, incidence of death due to suffering from HPV-associated cancers of different organs is 0.32 per 100.000 [10]. 
HPV is a double-stranded DNA virus that has an icosahedron shape and a doublelayered capsid made of 72 capsomers (HPV's capsid is not covered by a lipid membrane, which makes it resistant to ethanol and solvents.) It has 8,000 base pairs with a molecular mass of $5200 \mathrm{kDa}[8,11]$. The genes are divided into two groups: early (E) and late (L). The group of early genes is composed of six genes $(E 1, E 2, E 4, E 5, E 6, E 7)$ that code for the proteins responsible for replication, transcription, and malignant transformation $[5,8,11]$. Late genes $L 1$ and $L 2$ code for the viral envelope (non-lipid membrane) and for the proteins responsible for the capsid's structure. Gene $L 1$ is the oldest, and it is used to identify the different types of HPV. A $10 \%$ difference in the nucleotide sequence of the $L 1$ gene signifies a new strain (type), while a difference of $2-10 \%$ is considered a subtype, and a difference of less than $2 \%$ is defined as a variant.

The oncogenic potential of HPV depends on genes E6 and E7. The product of the E6 gene binds to the p53 oncosupressor, and the product of $E 7$ binds to the RB protein. In the case of cervical intraepithelial neoplasia, genes of high-risk types are integrated into the DNA of the host. This integration in some cases leads to disruption of the $E 2$ gene, which results in increased replication of the $E 6$ and $E 7$ genes. The E6 protein binds to p53 causing it to degrade. The E7 protein inactivates the RB protein so that E2F proteins detach from the $\mathrm{RB}$, preventing transcription of the gene that regulates cell growth and differentiation [5, 11]. It has been established that $\mathrm{E} 6$ and $\mathrm{E} 7$ interfere with the immune response by reducing production of interferon (IFN) [5]. Therefore, the E1 and E2 genes of HPVs are involved in viral replication, while the E6 and E7 proteins function as the promotors of proliferation. The major HPV oncogenes are E6 and E7, which disrupt the normal regulation of the cell cycle and cell progression, giving them an important role in the oncogenesis of HPVs with a high risk of causing anogenital and cervical cancer. Immortalization of epithelial cells induced by HPV requires viral DNA integration into the host cell genome, which causes disruption of the E2 gene. The E2 protein is also a transcription factor, which regulates expression of the E6 and E7 oncoproteins. Integration of the virus in the human genome disrupts the $E 2$ gene and increases expression of $E 6$ and $E 7$ genes in vitro [12-21].

Concerning molecular events during the progression of cervical lesions to carcinogenic lesions, persistent high-risk HPV infection leads to integration of HPV into the host genome and to overexpression of oncogenes E6 and E7 [11]. On the molecular level, interaction of E7 with the pRb protein leads to aberrant initiation of the S-phase. The E7 oncoprotein causes release of E2F transcription factor from the $\mathrm{pRb}$ protein, which is then active and can initiate transcription of genes involved in cell cycle progression, contributing to cellular immortalization and transformation. Thus, E6 targets p53 for proteasomal degradation, which leads to inhibition of apoptosis and DNA repair (anti-apoptotic effect). It is important to emphasize that only high-risk HPV types can induce degradation of p53, which can then lead to carcinogenesis. E6 activates the PI3K/Akt pathway, interacts with cellular proteins NFX1, and induces human telomerase reverse transcriptase ( $h T E R T$ ) activation, leading to immortalization and transformation. The interaction of both oncoproteins with DNA methyl transferases leads to aberrant methylation, causing silencing of tumor suppressor genes. Also, E7 interaction with histone deacetylases (HDACs) causes chromosome remodelling and genome instability. So, during lesional progression to carcinogenesis, the cross interaction of E6 and E7 with various pathways plays the crucial role [22]. It is also important to mention that viruses like HPV can create virions and become transmissible at any point in their life cycle (the productive virus replication also known as lytic replication). When lytic replication of the virus begins, it is almost irreversible, and successful replication of the virus begins as well as host cell death. But in tumor cells, these infections are mostly latent, allowing the virus to evade the immune response. Thus, lytic replication of the virus is reduced or absent in the tumor. In viral latency there in no production of unnecessary viral proteins that could initiate cell mediated immune recognition. Integration of the viral genome into the host genome eliminates the virus's ability to replicate as virions, but the virus can replicate using the host's cellular mechanisms and can be divided whenever the host cell divides. By 
evading apoptosis, as previously described, oncogenesis begins [23]. All this indicates that a complex network of actions is involved in the pathogenesis process during the occurrence of HPV manifestations.

\section{Stress, types of stressors, the hypothalamic-pituitary-adrenal (HPA) axis, the sympathetic autonomic nervous system (ANS) and the impact of stress on the body and disease}

Stress is defined as physical or mental exertion caused by factors that change homeostasis, and it can be observed as an objective stimulus, as an organism's response to stimulus, or as a relation between a person and their surrounding environment. Stress is a state of threat to physical, psychological, and social homeostasis. More recent approaches define stress as any stimulus that causes sudden termination of ordinary activities, that is, an event that goes beyond what is normal for the organism. The impact or effect, which stress can have, depends on a person's genetic pool, experiences, and behaviors [24-28]. The biopsychosocial model of disease and health, for example, asserts that biological, psychological and social factors interdependently affect the course and outcome of disease (Fig. 1). Psychological stress has become an increasingly important factor in the course of disease, due especially to the circumstances of modern life [25].

Stressors can be physical, chemical, psychological and biological. Psychological and social strains are the most common stressors [25]. Stress can be categorized by duration (acute or chronic), relevance (avoidable/averted or unavoidable), and intensity (mild, moderate, or severe). Aside from stress caused by common life events, there are also big traumatic events such as war, a natural disaster, death in the family, job loss, etc. Psych trauma is a state of high-level stress that can result in posttraumatic stress disorder (PTSD) and can cause long-lasting health problems. In physical, chemical, and biological stress, the condition of the person is determined by the harm caused by an external stimulus, whereas in psychological stress, one's assessment of environmental dangers, threats or challenges is the important factor. Stress in humans usually manifests through physical symptoms (e.g. palpitations, shortness of breath, perspiration, angina pectoris, frequent infections) or psychological symptoms (e.g. indecisiveness, poor concentration and memory loss, high sensitivity, sleep

Fig. 1. Factors which participate in onset and course of HPV manifestations (based on current literature data)

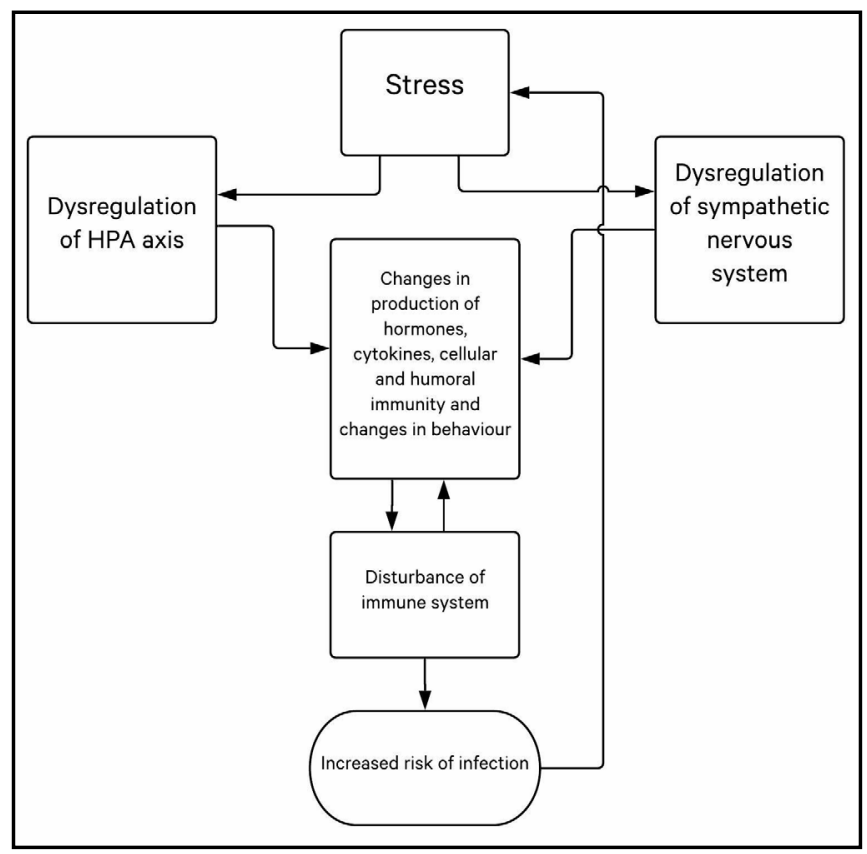


disturbances, negative thoughts). As a result, stress-related illnesses can occur (e.g. gastric ulcer, hypertension, viral infection, myocardial infarction, psoriasis, allergies, asthma, anxiety disorders, tumors, gastrointestinal disorders) [26-28].

When stressed, the organism reacts in a stress-adapting manner that can take place at the cell-, organ- or organ-system level, or at the level of the entire organism [25]. The organism typically reacts in three phases to a threat or injury with the same set of reactions outlined by the General Adaptation Syndrome (GAS): (1.) alarm; (2.) resistance; and (3.) exhaustion, the long-lasting debilitating phase that makes one susceptible to disease onset [29]. When reacting to external and internal demands by modulating functions and adapting to new conditions (referred to as alostasis), system stability can be achieved through constant adaptation. The main adaptive system includes the hypothalamic-pituitary-adrenal (HPA) axis, sympathetic autonomic nervous system, and cytokine production (Fig. 2) [1922]. Pathogenetically, the body neutralizes stress with a complex network of physiologic and behavioral responses to reestablish optimal body equilibrium (eustasis) [30]. As crucial components of the stress response, the HPA axis and the autonomic nervous system (ANS) interact with other vital centers in the central nervous system (CNS) and peripheral tissues/organs to mobilize an adequate/appropriate adaptive response against stressors. Thus, different stressful events are recognised by the hypothalamic paraventricular nucleus, which participates in a biological circuit that integrates personal experiences, physiological signalling and the release of corticotropin-releasing hormone (CRH) [31]. CRH acts on the pituitary gland, which then releases adrenocorticotropic hormone (ACTH), followed by ACTH signals to the adrenal cortex to release glucocorticoids [31]. Thus, the body's adaptive stress response depends on many interconnected neuroendocrine, immune, cellular, and molecular mechanisms.

During stress, the brain and CNS are the main actors; their response includes a variety of crucial neuroendocrine and autonomic reactions in order to achieve homeostasis [25-28, 3235]. During that process, neurogenic stressors activate processes in the CNS. Signals are then transferred to periventricular nuclei from prefrontal cortex and limbic structures, where stress is compared to experiential events. This processed signal is transferred to the hypothalamus, which in turn activates the HPA axis. Hypothalamic nuclei receive stimuli from limbic and

Fig. 2. Stress-induced reactions and psychoneuroimmune network during HPV onset, manifestations and course

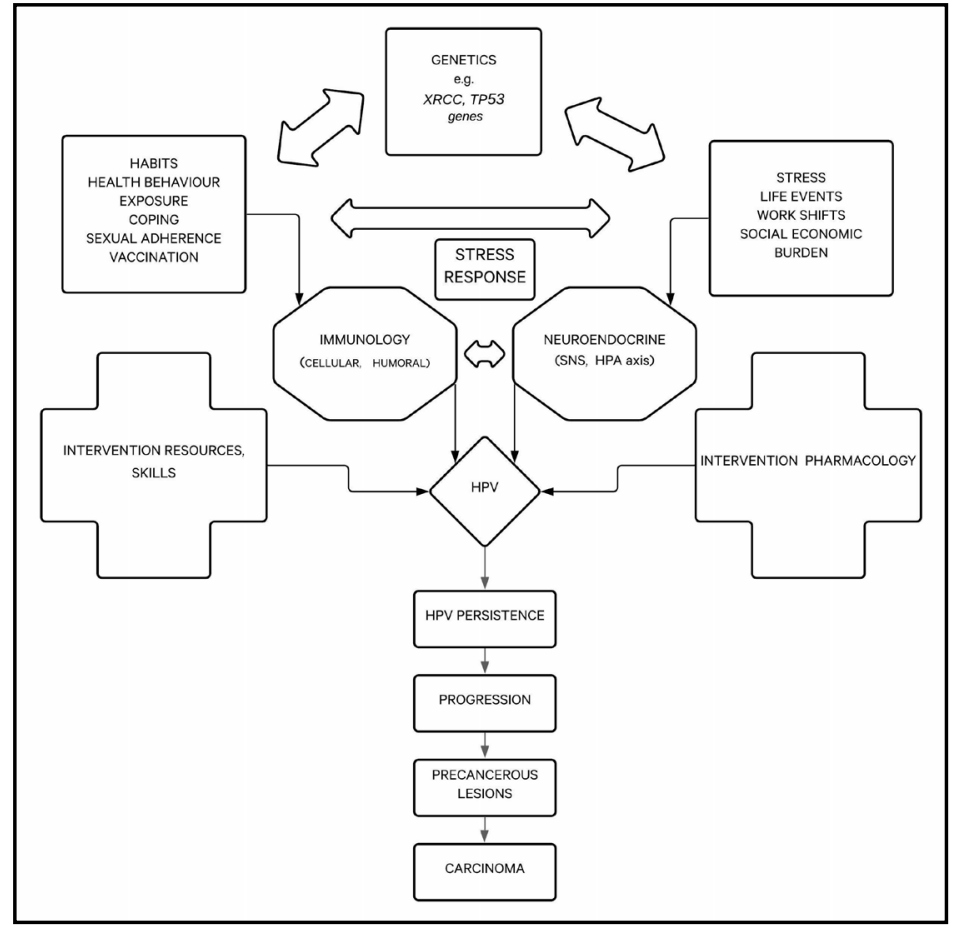


brain stem catecholaminergic signalling pathways. Periventricular nuclei can be activated by locus ceruleus aminergic signals. Central, medial and cortical amygdala nuclei are connected to periventricular and gabaergic neurons forming a closed circle. Activation of glutaminergic neurons stimulates the hypothalamic release of CRH in the eminentia mediana, which then reaches the hypophysis (anterior pituitary) through portal circulation and stimulates the release of ACTH into the peripheral circulation. Activation of the sympathetic nervous system causes terminal nerves and adrenal gland medulla to secret more catecholamines $[36,37]$. The immune system affects the brain as well; thus, there is a bilateral connection. It is important to emphasize that ACTH stimulates the release of glucocorticoids from the adrenal glands, meaning cortisol is a main stress hormone. Sympatho-adrenomedullary axis (SAM) activation stimulates CRH secretion in the hypothalamic periventricular neuron (PVN) area [25-28, 38]. Stress enhances the activities of many systems and releasing of various substances, including catecholamines, opiates and corticosteroids, which have an immunosuppressive effect by decreasing activity of cytokines and inflammation. It has been shown that immune cells have hormone receptors (corticosteroid, prolactin, growth hormone, sex hormones), neuropeptide receptors (endorphins, vasoactive intestinal peptide, substance P, etc.) and neurotransmitter receptors (adrenaline, noradrenaline, acetylcholine, serotonin, etc.). During acute stress, posterior hypothalamic nuclei, the sympathetic nervous system and adrenal gland medulla are activated, while in chronic stress, the anterior hypothalamus, sympathetic system and adrenal gland cortex are activated.

Finally, the release of glucocorticoids, mainly cortisol, is followed by increased lipolysis and gluconeogenesis to supply the body with available energy sources. A negative feedback system regulates production of cortisol via the hypothalamus and pituitary glands. In addition, the sympathetic nervous system (SNS) is activated and stimulates the adrenal medulla to release catecholamines, adrenaline and noradrenaline, allowing the body's systems to transport energy to the organs more quickly. Consequently, homeostasis is re-established, provided the stressor falls into the adaptive capacity [31]. However, during severe and/ or chronic stress, dysregulation of the stress system (hyperactivation or hypoactivation) can disrupt homeostasis and lead to cacostasis or allostasis with possible various clinical manifestations [30]. Prolonged activation of stress mechanisms with increased glucocorticoid and catecholamine levels causes a condition where the demand on the individual exceeds their personal adaptive capacity (allostatic load). Therefore, according to research results, chronic stress and increased glucocorticoid/catecholamine levels may participate in cancer progression in different diseases, including HPV-related carcinogenesis [31].

It is significant that cortisol, as the main stress hormone, modifies apoptosis and changes the way in which cytokines are secreted. It is assumed that exposure to relevant stress events can result in dysregulation of the sensitivity and numbers of glucocorticoid receptors (GCR), and that cortisol can affect secretion of local cytokines and trigger stronger inflammation [25, 27, 39]. Glucocorticoids exert their effects through two subtypes of intracellular receptors: (type I) GCR with a high affinity for endogenous corticosteroids (that has a regulating function over the circadian rhythm of the HPA axis) and (type II) GCR with a lower affinity (important for acute stress reactions). In the nucleus, glucocorticoid acts as a transcription factor, and it binds to specific DNA sequences named glucocorticoid response elements (GRE). These sequences modulate gene transcription. Glucocorticoids also interact with other transcription factors, such as AP-1 (activator protein 1) and NF- $\mathrm{kB}$ (nuclear factor kappa $B$ ). During a stress inflammatory response, glucocorticoids decrease secretion of proinflammatory cytokines and increase secretion of anti-inflammatory cytokines. They also affect redistribution of leukocytes and decrease synthesis and expression of cytokine receptors, lymphocyte proliferation and adhesion molecule expression on the cell surface.

Finally, in stress, adaptive reactions involve the short-term activation of the HPA axis, whereas overproduction of stress hormones and disruption of the regulation of the HPA axis triggers a pathological response/reaction. Just as the cognitive perception of stress is important, so is coping, defined as the constant adaptation of cognitive and behavioral efforts to overcome demands that one finds overwhelming. Adaptive coping styles are 
usually related to positive personality characteristics, while maladaptive styles are linked to less desireable characteristics. The coping styles a person uses depend primarily upon the situation but also on the disposition of the person themselves.

\section{Results}

The most important results and current knowledge on HPV, stress and carcinogenesis will be presented here, and relevant trends and patterns will be described.

\section{Psychological stress and immunological parameters related to infections}

Research in the field of acute and chronic stress have shown that many biochemical changes, e.g. changes in the blood, have been observed in people experiencing stressful events. For example, acute stress increases the number of lymphocytes in the peripheral blood, especially natural killer (NK) cells. Also, during short-term stress events (e.g., an exam), cytokine production disturbances have been observed along with a shift from $\mathrm{T}$ helper lymphocytes type 1 (Th1) towards T helper lymphocytes type 2 (Th2), with higher levels of immunoglobulins [25-28,38].

During chronic stress, lower numbers of lymphocytes, decreased NK cell activity, lymphocyte proliferation to mitogen stimulation and phagocytosis, decreased cellular immunity, and late hypersensitivity reactions were observed. According to previous research results, during psychological stress, higher titers of antibodies against viral infection (Epstein-Barr virus antibodies) as well as reactivation of EBV, cytomegalovirus, and herpes simplex virus were recorded [25]. Thereby, latent viruses can be reactivated, and antibody production decreased. In the setting of chronic stress, there is a shift towards a Th2 response (with limited production of IFN and IL-2), which enhances humoral immunity and decreases cellular immunity, possibly affecting the development of infections and autoimmune and malignant diseases. Immune responses to stress depend on duration of the stress factor effect; subsequently, stress of shorter duration causes translation from specific to innate immunity, while in the setting of chronic stress, this translation is seen in a change from a Th1 type reaction to a Th2 type reaction. It is significant that Th1 cells engage in cellular immunity, secrete IL- 2 and IFN- $\gamma$, TNF- $\beta$ and activate macrophages, while Th 2 cells induce humoral immunity by activating the B cell response and antibody production, as well as secreting IL-3, IL-5, IL-10, and IL-13.

Increased psychological stress can result in a higher occurrence of viral infections, especially those mediated by IL- 6 . The influence of stress on cell apoptosis can also be observed, where apoptosis disruption can lead to immunosuppression. For instance, in acute stress (e.g. academic stress) the process of apoptosis, important in carcinogenesis and the function of the immune system, is more frequently blocked. In a specific example, a connection has been observed between women with cervical lesions who have a pessimistic view of the world and immune system disorders, lower infection control and a higher risk of neoplastic cervical lesion progression. Chronically high sympathetic system activity decreases lymphocyte proliferation and changes the Th1/Th2 cell ratio in a way that suppresses Th1 cytokines, therefore reaction evolves in the direction of Th2 cells, which can increase the risk for a high virus replication rate.

Also, the relationship between psychological stress and increased sensitivity to respiratory infections can be explained by the impact of stressful life events on immunological functions. It has been shown that psychosocial stress and limited social support can have a big impact by increasing susceptibility to upper respiratory tract viral infections. Patients with colds, for example, had a statistically significant higher average sum of greater stressful life events in the previous year compared to patients without a cold [40]. Results have also concluded that urinary system infections are more frequent in people who suffer greater stress [41]. Concerning HPV, it has been observed that a higher number of life stressors in at least the previous 6 months, the absence of social support and the types of personal coping 
mechanisms employed influenced the progression of HPV infection [25, 42]. In women, it has been shown that HPV infection and stress result in the disruption of IL-6 secretion and the progression of cervical lesions to cervical dysplasia. In chronically stressed caregivers who care for relatives suffering from dementia, a fourfold increase in IL-6 concentration was seen (by longitudinal observation). In women with cervical dysplasia, a connection between greater stress experiences and dysregulation of specific immune responses has been observed.

The connection between stress, immune factors, infections and carcinogenesis often includes a link between HPA/SNS functions, immunosuppression and viral oncogenesis, which together work within the immune response - both glucocorticoids and catecholamines regulate different immune functions (antigen presentation, T-cell proliferation, cell-mediated reactions, humoral immunity, etc.) [43-45]. Stress-induced immunosuppression is especially important for infection-related cancer, whereas disturbed cellular immunity may contribute to increased risk of oncogenic viral infection and DNA damage. Since several human tumor viruses including HPVs (HPV 16, HPV 33, etc.) are sensitive to the glucocorticoid/ catecholamine-activating signalling pathways, stress hormones may consequently reactivate latent tumor viruses, stimulate viral oncogenes expression, and inhibit antiviral host responses. In response to glucocorticoids, high-risk HPV may activate gene expression, interact with cellular proto-oncogenes and evade the cellular immune response by downregulating the HLA expression of class. Conversely, glucocorticoid antagonists suppress infection (the HPV activity) [43].

Pathogenesis of HPV infection and HPV-related carcinogenesis and possible association/ relation with stress and psychosocial factors

Once HPV enters a cell via the $\alpha 6$ integrin (which functions as a receptor) there are three possible sequences: latent infection (identified only by molecular diagnostic methods), subclinical infection (identified by colposcopy, peniscopy or microscopic examination), and clinically manifest disease. Incubation lasts 1 to 8 months [44]. HPV proliferation in differentiated epithelial cells induces morphologically cytopathic changes in the form of koilocytosis best seen in condyloma (koilocytosis is not specific to HPV) $[44,46]$. HPV infection histologically manifests with thickening of the epidermis, spinous layer, hyperplasia, and hyperkeratosis. Another important pathogenic factor for HPV lesion ocurrence is ultraviolet light (UV). Normally, UV induces mutations in basal keratinocytes with consequent repair of DNA mutations or apoptosis. In the case of HPV infection, the cellular response to UVinduced damage is inhibited by E6 and E7 expression. This causes proliferation of damaged cells with a potential for malignant alteration [1].

Aside from the skin, HPV DNA can be found in anogenital and oropharingeal malignancies as well as precancerous conditions. In these tissues' viral oncogenes, E6 and E7 expression is regularly present. Expression of the $E 6$ and $E 7$ genes is also found in cervical cancer cells. All this supports the observation that HPV has a role in the etiology of epithelial cancer [8]. Furthermore, epidemiology data suggest that HPV infection is a major factor in the etiology of cervical cancer. For the formation of irreversible malignancy, the following sequence is necessary: initial expression of $E 6$ and $E 7$ genes followed by suppression of apoptosis and the stabile expression of E6 and E7 proteins that protect transformed cells from apoptosis. This is also the reason for tumor resistance against chemotherapy or hypoxia in HPV malignant lesions $[8,46]$.

Changes in cellular immunity in HPV infection are associated with changes in cytokine levels, induction of cellular adhesion molecules, and changes in molecules of tissue histocompatibility, human leukocyte antigens (HLA). Thus IFN- $\gamma$ and cytokines act on the JAK signal transduction pathway. In vitro studies indicate that E region of HPV can bind to the signal transducer and activator of transcription (STAT) protein and inhibit IFN genes. E6 also reduces IFN gene expression and binds to Tyk2 kinase, preventing STAT protein phosphorylation and reduced cellular response to infection. It has also been found that up to $60 \%$ of HPV-infected patients have a low titer of antibodies that are specific for the viral capsid [47]. 
Psychosocial factors may affect the development and persistence of HPV, primarily by acting on the immune response. An association between psychosocial stress and CIN development in women has been established. HPV has developed a number of ways to avoid hosts' responses to infection. It hides the expression of viral proteins and reduces the expression of genes for IFN- $\alpha$ in keratinocytes. A successful immune response is characterized by a strong, local cell-mediated immune response. The immune response depends on the duration of stressors-a shorter duration of stress shifts from specific to innate immunity, while chronic stress shifts it from the Th1 type of reaction to the Th2 type of reaction. Significantly, Th1 cells participate in cellular immunity, secrete IL- 2 , IFN- $\gamma$, and TNF- $\beta$ and activate macrophages, and Th2 cells stimulate humoral immunity, activate the B cell response and antibody production, as well as promote the secretion of IL-3, IL-5, IL-10, and IL-13. The natural course of HPV infection can vary. It may disappear spontaneously, last, or lead to the appearance of intraepithelial neoplastic lesions and, ultimately, to the appearance of cancer. Oncogenic transformation requires the integration of the virus genome into the host genome. In doing so, DNA in the E1 region of E2 breaks down, leading to transcription disorders of E6 and E7. A number of other risk factors are also involved in the onset of malignant transformation: promiscuity, a lower socioeconomic status, a higher number of pregnancies and abortions, use of oral contraceptives, smoking, co-infections, and stress. The pathogenetic life cycle of HPV can be divided into two stages: (stage 1) persistence, i.e. HPV DNA is found in basal cells lasting from a few weeks to several years, with a low level of virus replication over many generations) and (stage 2) the productive phase (occurs in terminally differentiated suprabasal keratinocytes with the appearance of viral DNA amplification, capsid gene expression and virion formation) [44]. Several factors are important for the regression of HPV manifestation/infection, one of which is the influence of psychological stress, because it has been proven that stress can prolong the duration and severity of HPV disease [25]. In the regression of HPV infection, increased activity of Th1 cells was observed. However, during psychosocial stress, a decrease in the Th1 type of immune response is seen, and there is a shift towards the Th2 type of immune response.

The association between psychologic stress and HPV carcinogenesis can be explained by a few possible mechanisms [43]. The stress-activated HPA axis and SNS act through glucocorticoid- and adrenergic- pathways which impact immune regulation, involving stressinduced immunosuppression and oncogenic infection and other potentially stress-triggered neuroendocrine transmitters which influence cancerogenesis (e.g. serotonin promotes tumor cell growth and angiogenesis; dopamine exerts opposite actions) [43]. The adrenergic pathway and SNS activation support carcinogenesis through tumor growth, malignant transformation, macrophage infiltration, angiogenesis, inflammation, dissemination, etc. Thus, in cervical cancer cells, sustained adrenergic signalling results in protein kinase A (PKA) activation, which then causes inhibition of the tumour suppressive pathway (Hippo Yap pathway). According to research data, a strong association between stress/bereavement and a high viral load infection (HPV16) includes the promoting of overexpression and malignant transformation of HPV oncogenes, and a strong association between repeated infections and bereavement/stress may compromise host immunosurveillance and mediate HPV persistence or reactivation [45]. According to Lu, stress/bereavement was associated with an increased risk for cervical cancerogenesis, especially for women with high screening adherence or when multiple losses, loss of a child, sibling or spouse, and loss due to unnatural causes were analyzed separately [45]. Thus, stress hormones (corticosteroids) affect signal pathways involved in the malignant cell transformation processes induced by oncogenic human viruses. 


\section{Discussion}

It is known that stress affects the immune system by redistributing immune cells (leukocyte and neutrophil numbers increase, lymphocyte numbers decrease), which is supported by increased activities/values of catecholamines, cortisol, chemokines and adhesion molecules secreted during stress reactions [48-52]. Leukocytes are especially important because they secrete cytokines, affect the HPA, and have cytokine, hormone, neurotransmitter and growth factor receptors, etc. Current studies have observed that stressful life events can influence genes. That is, they exert activity changes in a variety of genes, which then leads to modulation of immunological reactions, including leukocyte numbers and distribution [52]. Furthermore, it has been seen in animal models that the neutrophil to lymphocyte ratio predicts susceptibility to infection [48]. A higher cortisol level, combined with increased neutrophil and decreased lymphocyte numbers, has been observed in psychologically stressed people [25]. There are some observed (biochemical) changes in plasma protein levels, as confirmed by studies of their values during illness and stress. There are various study results on animals and humans which support the association between psychological stress, infections and carcinogenesis (Table 1 and Table 2) [25, 48, 53-70]. Animal model studies show that stress increases values of acute phase proteins [71]. After stress, haptoglobin significantly increases, so it is used as a stress marker during animal transport and for evaluation of the animal's condition [72]. During acute academic stress, elevated total levels of serum proteins, alpha- 2 and beta and gamma globulins, have been observed [73]. Similar results are seen in research by Cvitanović, where elevated leukocytes, alpha-2 and beta globulins were observed among HPV patients with significant experienced stress [25]. Immunocompromised patients are more susceptible to developing a variety of infections, including HPV infection.

Research results in the field of hormonal changes in stressed people, especially changes in cortisol levels and hormones of the HPA axis, are significant as well. Hypocortisolemia is evidenced in people subjected to severe stress, as well as in chronic fatigue syndrome, fibromyalgia, and rheumatoid arthritis. On the other hand, elevated glucocorticoid levels are associated with elevated leukocytes, decreased numbers of circulating lymphocytes and a decreased neutrophil to lymphocyte ratio. Cole has observed a significant correlation

Table 1. Prominent animal study results which support the association between psychological stress, inflammation and carcinogenesis (articles are in alphabetical order by author)

\begin{tabular}{|c|c|c|c|}
\hline Author & Animals/Subjects & Methods and type of study & Main results and conclusions \\
\hline $\begin{array}{l}\text { Armaiz-Pena GN, } \\
2015\end{array}$ & Mice & $\begin{array}{l}\text { In mice subjected to daily restraint stress, factors responsible for } \\
\text { stress-mediated effects on monocyte/macrophage recruitment into } \\
\text { the tumor microenvironment, and consequent effects on tumor } \\
\text { growth, were examined. }\end{array}$ & $\begin{array}{l}\text { In stressed mice, tumor samples showed elevated MCP1 gene and protein levels, increased CD14+ cells, and } \\
\text { increased infiltration of CD68 cells. In ovarian cancer of animals, elevated peripheral blood monocytes and } \\
\text { tumoral macrophages were linked to worse rates of survival overall. Increased adrenergic signaling is associated } \\
\text { with macrophage infiltration and mediated by tumor cell derived MCP1 production. }\end{array}$ \\
\hline $\begin{array}{l}\text { Jensen MM, } \\
1968\end{array}$ & Mice & $\begin{array}{l}\text { Infected mice (viral infection) were subjected to sound or } \\
\text { avoidance-learning stress. }\end{array}$ & $\begin{array}{l}\text { Stress applied before viral injection did not impact spleen size, while stress applied after infection markedly } \\
\text { retarded splenic enlargement. This phenomenon was not seen in adrenalectomized mice. Stressed mice showed } \\
\text { slightly lower death rates. }\end{array}$ \\
\hline $\begin{array}{l}\text { Magnon C, } \\
2013\end{array}$ & $\begin{array}{l}\text { Mice (mouse } \\
\text { model) }\end{array}$ & $\begin{array}{l}\text { Analysis of prostate gland cancer for densities of tumoral } \\
\text { sympathetic and parasympathetic nerve fibers according to clinical } \\
\text { outcomes. }\end{array}$ & $\begin{array}{l}\text { The formation of autonomic nerve fibers regulates prostate cancer development and dissemination. } \\
\text { Sympathectomy or genetic deletion of stromal } \beta 2-/ \beta 3 \text {-AR prevented the early phases of tumor development. } \\
\text { Pharmacological blockade or genetic disruption of the stromal MR1 blocked tumor invasion and metastasis. } \\
\text { Higher densities of sympathetic and parasympathetic nerve fibers in tumor and surrounding tissue were } \\
\text { associated with poor clinical outcomes. }\end{array}$ \\
\hline $\begin{array}{l}\text { Powell ND, } \\
2013\end{array}$ & Murine model & $\begin{array}{l}\text { Review and analysis of different studies (animal, human) on } \\
\text { psychological stress-impact mechanisms (sympathetic, } \\
\text { neuroendocrine and immunologic) in cancer biology, including } \\
\text { social disruption (SDR) stress. }\end{array}$ & $\begin{array}{l}\text { Psychosocial stress triggers sympathetic and neuroendocrine responses, which significantly impacts cancer, in } \\
\text { part, through regulation of inflammatory mediators. }\end{array}$ \\
\hline $\begin{array}{l}\text { Hunzeker J, } \\
2004\end{array}$ & Mice & $\begin{array}{l}\text { The influences of restraint stress (RST) on natural killer (NK) } \\
\text { activity and its consequences on influenza A/PR8 (A/PR8) viral } \\
\text { replication were examined (analysis for lung) during an } \\
\text { experimental influenza viral infections. }\end{array}$ & $\begin{array}{l}\text { Restraint stress (RST) suppressed the chemokines responsible for NK cell recruitment into the infected tissue } \\
\text { and suppressed several macrophage-derived cytokines expressions in the effector response of NK cells } \\
\text { (important innate response to control viral replication) and also supressed Ll-15 (important for NK cell } \\
\text { development and homeostasis) and IL-12 (important for NK cytotoxicity). }\end{array}$ \\
\hline $\begin{array}{l}\text { Stefanski V, } \\
2003\end{array}$ & $\begin{array}{l}\text { Rat (a male } \\
\text { Fischer 344) }\end{array}$ & $\begin{array}{l}\text { In socially stressed male rat, the effects of confrontation stress on } \\
\text { the normal distribution of T cells into lymphoid organs and other } \\
\text { tissues were examined. }\end{array}$ & $\begin{array}{l}24 \text { hours after injection of } 51 \mathrm{Cr}-\text { labeled blood } \mathrm{T} \text { cells isolated from syngeneic donor, socially stressed male rat } \\
\text { showed lower accumulations of } 5 \mathrm{CCr} \text {-labeledd cells in lymphoid organs (the spleen, mesenteric and cervical } \\
\text { lymph nodes) (about } 30 \% \text { lower than control males). In the bone marrow of socially stressed males, substantially } \\
\text { higher localization was observed. }\end{array}$ \\
\hline $\begin{array}{l}\text { Thaker PH, } \\
2006\end{array}$ & $\begin{array}{l}\text { Mice (orthotopic } \\
\text { mouse model) }\end{array}$ & $\begin{array}{l}\text { Analysis of ovarian cancer cells (mouse model) for angiogenesis } \\
\text { and vascularization under stress. }\end{array}$ & $\begin{array}{l}\text { In stressed mice, ovarian cancer showed markedly increased vascularization and enhanced expression of VEGF, } \\
\text { MMP2 and MMP9, and angiogenic processes mediated the effects of stress on tumor growth. Behavioral stress } \\
\text { enhances tumor angiogenensis primarily through beta-adrenergic activation of the cAMPP-PAA signaling pathway } \\
\text { (crucial mechanism for promotion malignant cell growth). }\end{array}$ \\
\hline $\begin{array}{l}\text { Verza FA, } \\
2021\end{array}$ & $\begin{array}{l}\text { Rats (21-day-old } \\
\text { male Wistar rats) }\end{array}$ & $\begin{array}{l}\text { Social isolation stress effects on chemically induced oral } \\
\text { carcinogenesis were examined in rats (isolated and grouped } \\
\text { groups); after } 90 \text { d ays of age, the rats (both groups) underwent oral } \\
\text { carcinogenesis (with 4-nitroquinoline 1-oxide (4NQ0) for } 20 \\
\text { weeks) and were assessed for depressive-like behavior, then } \\
\text { euthanized for an oral squamous cell carcinoma (OSCC) diagnosis } \\
\text { and measurement of local tumor inflammatory mediators. }\end{array}$ & 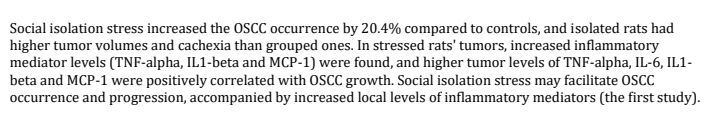 \\
\hline
\end{tabular}


Cell Physiol Biochem 2021;55(S2):71-88

DOI: $10.33594 / 000000395$

(C) 2021 The Author(s). Published by

\begin{tabular}{l|l} 
Published online: 10 July 2021 & Cell Physiol Biochem Press GmbH\&Co. KG \\
\hline
\end{tabular}

Lugović-Mihić et al.: Psychological Stress, HPV Infection and Carcinogenesis

Table 2. Main study results (human subjects) which support the association between psychological stress, infections and carcinogenesis. Abbreviations: Appr-approximately; ADRB- $\beta$-adrenergic receptor; HPV Human papillomavirus; PBI - psychosomatic behavioral interventions; RRs - relative risks; SIL - squamous intraepithelial lesions; TCM - traditional Chinese medicine

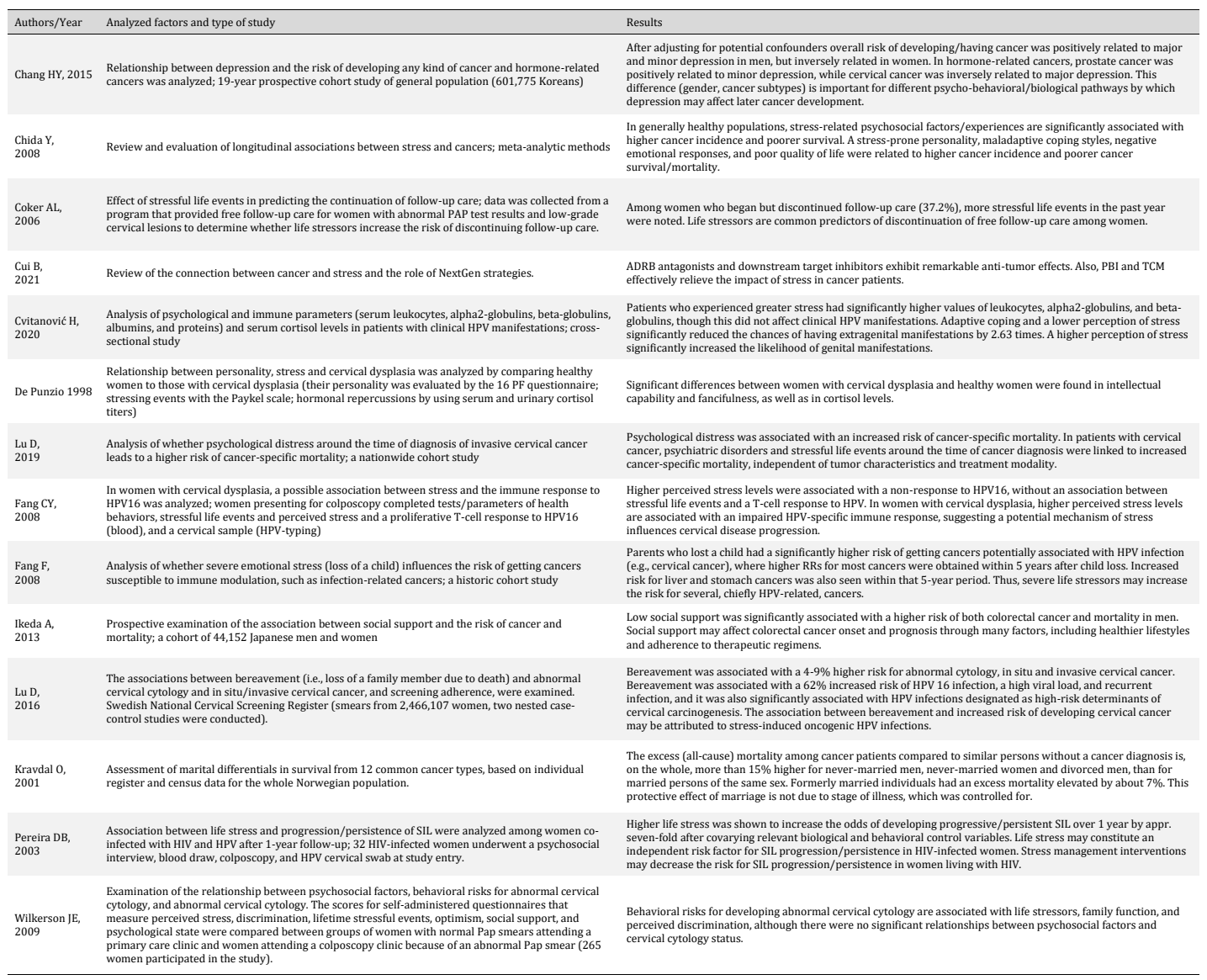

between circulating leukocyte numbers and cortisol concentration only in the presence of GCR sensitivity [74]. Observations on the possible mechanism connecting chronic stress and infection are considerable. People who are chronically stressed throughout life could be at risk for an HPA disorder and decreased immune defense reactions to infections, which are mediated by many cytokines and other transmitters. When cortisol regulation and cytokine production are disturbed, a disbalance of the Th1/Th2 immune reaction and the emergence of clinical symptoms, are possible. Subsequently, in patients exposed to severe chronic stress, a weakening of the HPA axis is observed $[74,75]$. There are specific examples of cortisol levels being connected with immunological changes, although results are not always consistent. According to some studies on HPV infection, there is a negative correlation between patient cortisol levels and exposure to chronic stress; other studies show no such correlation [13]. However, there are more results showing that higher cortisol levels and higher levels of experienced stress are connected to the progression of CIN changes

Previous studies on corticosteroids in patients with various cancers indicated association/relation [31, 43, 55]. For example, in patients with prostate cancer, cortisol stimulates carcinogenesis by activating the androgen receptor in the absence of androgens. In ovarian cancer, glucocorticoids have anti-apoptotic effects on cancer cell lines, by upregulation of the anti-apoptotic proteins. Also, in colon cancer, the stress hormones pathways induce the proliferation of metastatic cancer cells by upregulating CDK1 expression (a dysregulation of CDK is associated with tumour proliferation activities). Studies on cervical 
and lung cancers showed that dexamethasone influences cell apoptosis. Dexamethasone downregulated cisplatin-induced expression of cell death receptor pathway components, which indicates its anti-apoptotic role in many cancers, although pro-apoptotic actions are seen in lymphoid carcinomas. Also, glucocorticoids activate the PI3K/AKT pathway which inactivates pro-apoptotic factors/molecules (eg. caspases). This is the same pathway that HPV uses to cause immortality of transformed cells. HPVs E6 protein can activate oncogenic pathways including phosphoinositide 3-kinase (PI3K)/protein B (Akt), Wnt and Notch. The PI3K/Akt pathway controls cell proliferation, cell growth, angiogenesis and cell survival by influencing multiple downstream targets. In addition, dexamethasone increases some cancers' resistance to cytotoxic therapy (hepatocellular and colorectal), and in some cancers dexamethasone enhanced the growth of cell lines treated with 5-fluorouracil or cisplatin (breast, cervical, melanoma, neuroblastoma). For oncology patients, it is important to consider that glucocorticoids may induce chemotherapy resistance by blocking tumor cell death (i.e. anti-apoptotic effect), although cortisol is routinely administered throughout chemotherapy for treatment/prevention of nausea and allergic reactions. Corticosteroids affect signal pathways involved in the malignant cell transformation processes induced by oncogenic viruses. Glucocorticoid signaling participates in solid tumor progression through increased cell proliferation, inhibited apoptosis and DNA repair activity. In addition, in many cancer patients, chronic stress is associated with flattened diurnal cortisol rhythms and increased nocturnal cortisol, especially in those in advanced disease/cancer stages, and the altered cortisol patterns may partially reflect patient distress and/or avoidant coping. In various cancers (breast, lung, renal), the flattened cortisol slope is associated with compromised survival [43].

Not only are there studies that show the influence of psychological stress on immunological parameters and infection and carcinogenesis, but it has also been suggested that psychological stress impacts clinical manifestations of HPV, though study results are not consistent $[25,45,68,70,76-78]$. In several studies, the influence of negative life events, specifically, on clinical manifestations of HPV has been researched. Also, Pereira has observed that at higher levels of exposure to stress there is progression and persistence of precancerous HPV lesions [69]. According to Coker, psychosocial stress influences the course of genital HPV infection. Some other recent studies also show a connection between infections and stress [62]. It has been shown that perceived stress is associated with a longer duration of HPV infection in men over 50 years of age, although the association with HPV infection incidence has not been confirmed. There is some evidence that level of perceived stress (but not the number of negative life experiences) is associated with unreactivity to HPV 16, which was explained by the Th1/Th2 balance shift towards a Th2 type cytokine reaction $[51,78]$. Coker also demonstrated that in Caucasian women, negative life events caused deterioration of HPV lesions [62]. The same was not true for Afroamerican women, which was explained by socioeconomic differences. Furthermore, Coker has demonstrated that psychological abuse is associated with pathological findings in Papanicolau cervical smears.

Unlike Coker, Wilkerson did not find any correlation between pathological findings of clinical HPV manifestations and perceived stress or number of negative life events [70]. Likewise, Sharp did not find a significant correlation between the influence of negative life events and HPV lesions [76]. Also, according to Massad, there is no statistically significant correlation between perceived stress and clinical HPV manifestations [79]. According to research by Massad and research by Wilkerson, it hasn't been determined whether stress or depression affects the prevalence of changes associated with HPV [70, 79]. Still, most research points to stress being a potential risk factor for development of HPV infection [51, $68,80]$. The influence of stress on patient compliance, and eventually on development of HPV skin and mucosal lesions, should also be considered because elevated stress levels can lead to decreased patient compliance, thus persistence or progression of intraepithelial lesions [70]. 
There are also some differences by sex in how stress affects HPV manifestations [25]. Thus, it has been observed that men, after being exposed to psychological stress, are more vulnerable to diseases than women. Longer lasting HPV infection in women who are older and have more notable stressful experiences was also observed. There is also a difference between the sexes in regard to localization of HPV manifestations-in women lesions in the genital region are more common, while in men they are more common in the oral region. Also, HPV transmission rates are higher in women, and the immune response, which is stronger in women, depends on anatomic localization. It has been observed that women are better protected from infection/the effects or manifestations of infection. Another difference seen in men is a lower prevalence of HPV 6,11, 16 and 18. Men and women also perceive the importance of HPV infection differently by the very fact that there is a much higher incidence of carcinoma in women with evident HPV infection. There is an added impact of socioeconomic status on HPV manifestations for women of lower socioeconomic status, for whom, even though they don't have more prevalent HPV infections, carcinoma caused by HPV is more prevalent. This is presumably due to decreased accessibility to health resources, but also due to greater stress, maladaptive coping styles and limited social support.

With HPV infection, the patient's perception of stress is important [80-84]. According to research by Cvitanović, a higher level of perceived stress means the probability of genital manifestations significantly increases (3.53 times) [25]. McCaffery demonstrated similar results, and Coker also observed that positive HPV testing results lead to increased anxiety $[62,82]$. Also, a higher level of perceived stress has been demonstrated in women with cervical dysplasia and HPV. Increased lytic expression of HPV as a source of stress is also an important concern. A woman might become stressed simply by the appearance of changes on the cervix. This could lead to greater anxiety and the possible progression of those changes/ lesions. Thus, a patient's psychological state can lead to a closed cycle of progession of the manifested disease [85].

Thus, adaptive coping mechanisms for dealing with stress, including stress caused by disease, became a crucial part of avoiding a variety of health problems. According to some research on women, stress and the process of coping with disease can decrease immunity and thus increase the risk for clinically relevant HPV manifestations. Antoni observed a higher incidence of cervical dysplasia in women with maladaptive coping styles than in women with adaptive coping styles [80]. Other research has been done on the association between psychological changes and cervical carcinoma, particularly looking at stressors and stressful events that subjects have little control over. That kind of stress is considerably associated with pathological findings of squamous intraepithelial lesion (SIL). Abuse (in childhood or by a partner) is also associated with pathological findings of SIL. Psychological stress and maladaptive coping styles are also associated with a higher risk of SIL and invasive cervical carcinoma development, which is explained by decreased immune function. It has also been observed that changes in the form of SIL are more frequent in persons suffering from alexithymia, in whom decreased numbers of lymphocytes have been demonstrated. A relationship between infection and psychological factors has also been recorded, e.g. in anxious women, cervical cancer was more commonly found than endometrial cancer (which is not etiopathologically associated with infection). Regarding all of this, it should be emphasized that certain methodological issues arise when investigating the association between stress, the immune response and disease. Tests that investigate the influence of stress on immunological parameters are quite variable and depend upon many nonimmunological factors (age, sex, diet, smoking, substance abuse, lifestyle and socioeconomic status).

Research literature has proposed possible psychological treatments for addressing patients with HPV $[25,70,76,78,79]$. Antoni asked female patients with clinically manifested HPV infection in the form of a CIN to undergo cognitive-behavioral therapy and observed that those who underwent treatment experienced substantially less frequent occurrences of severe lesions with higher stages of CIN [80]. This suggests that including stress-management in standard dermatological practice could be effective, especially for patients suffering from anogenital HPV lesions [79]. By using adaptive methods of coping with stress and raising 
awareness of the effect a patient's perception of stress can have on disease manifestations, patient quality of life can be significantly improved and allostatic burden can be diminished.

Chronic stress may negatively influence patients with cancers through suppression of protective immunity related with cancer exacerbation and progression or metastasis, which is important for their immuno-protective responses and successful oncology treatment (primarily tumor immunotherapy), especiall for virally-associated cancers (HPV-associated cervical, anal, and oral cancers etc.). While previous research has thoroughly examined the effects of stress on some cancers (breast, prostate, lung, ovarian), more data for other cancers could be collected and examined in greater detail. Limited data on the stress-HPVcarcinogenesis association indicates a need for more research on the different features/ mechanisms of stress-induced cancer progression. Since stress hormones and immune disturbances may affect carcinogenesis, there is a need to expand current knowledge on stress signalling in cancers (e.g. HPV carcinogenesis) and for a more precise understanding of stress. Multidisciplinary approaches to research and therapy including diverse sets of participants and factors/effects related to stress would be greatly beneficial. Also, it is necessary to better understand psychological/psychosocial features of cancer patients, e.g. how stress influences underlying molecular mechanisms. According to current knowledge, there are various possible ways to relieve the impact of psychological stress on cancer patients, such as the use of $\beta$-adrenergic receptor (ADRB) antagonists and downstream target inhibitors (which have significant anti-tumor effects), psychosomatic behavioral interventions, and traditional Chinese medicine [63]. Since the influence of psychological stress on HPV infection manifestations and carcinogenesis has been proven by research, specific psychological health measures and treatments should be designed and implemented to help patients deal with stress and its adverse effects in a healthy way.

\section{Conclusion}

Present findings suggest that stress is an important risk factor for HPV manifestation development and carcinogenesis. Understanding the key factors and processes clears the way for effective prevention and therapeutic intervention. Even though psychological factors have not been considered in many past epidemiological studies, they are now understood to be important in the setting of frequent and sometimes very serious illnesses such as HPV. Understanding perceived stress and biological changes in stress, as well as the evaluation of immune parameters, gives researchers a better picture of how stress influences HPV infections and how to improve disease management and outcomes.

\section{Acknowledgements}

We would like to express our heartfelt gratitude to Mrs. Suzana Salopek for her selfless help and valuable assistance.

\section{Author Contributions}

LLM contributed conception and design of the article; LLM, HC, MK and AŠ analyzed the data; LLM, HC and ID wrote the manuscript; all authors contributed to manuscript revision, read and approved the submitted version.

Statement of Ethics

The authors have no ethical conflicts to disclose. 


\section{Cellular Physiology Cell Physiol Biochem 2021;55(\$2):71-88 \\ \begin{tabular}{ll|l} 
and Biochemistry $10.33594 / 000000395$ & Published online: 10 July 2021 & $\begin{array}{l}\text { C } 2021 \text { The Author(s). Published by } \\
\text { Cell Physiol Biochem Press GmbH\&Co. KG }\end{array}$
\end{tabular}}

Lugović-Mihić et al.: Psychological Stress, HPV Infection and Carcinogenesis

\section{Disclosure Statement}

The authors declare that no conflicts of interest exist.

\section{References}

1 Tommasino M: HPV and skin carcinogenesis. Papillomavirus Res 2019;7:129-131.

2 Smola S: Human papillomaviruses and skin cancer. Adv Exp Med Biol 2020;1268:195-209.

3 Clifford GM, Tully S, Franceschi S: Carcinogenicity of human papillomavirus (HPV) types in HIV-positive women: a meta-analysis from HPV infection to cervical cancer. Clin Infect Dis 2017;64:1228-1235.

4 Stanley M: Immune responses to human papillomavirus. Vaccine 2006;24:S16-S22.

5 Palefsky JM: Human papillomavirus infections: Epidemiology and disease associations, 2020. URL: https://www.uptodate.com/contents/human-papillomavirus-infections-epidemiology-and-diseaseassociations?search=human-papillomavirus-hpv\&source=search_result\&selectedTitle=1 150\&usag e_type=default\&display_rank=1

6 Tyring SK: Human papillomavirus infections: epidemiology, pathogenesis, and host immune response. J Am Acad Dermatol 2000;43:18-26.

7 Graziottin A, Serafini A: HPV Infection in women: psychosexual impact of genital warts and intraepithelial lesions. J Sex Med 2009;6:633-645.

8 De Villiers EM, Fauquet C, Broker TR, Bernard HU, zur Hausen H: Classification of papillomaviruses. Virology 2004;324:17-27.

9 de Sanjosé S, Brotons M, Pavón MA: The natural history of human papillomavirus infection. Best Pract Res Clin Obstet Gynaecol 2018;47:2-13.

10 Duan R, Qiao Y, Clifford G, Zhao F: Cancer burden attributable to human papillomavirus infection by sex, cancer site, age, and geographical area in China. Cancer Med 2020;9:374-384.

11 Tjiong MY, Out TA, Ter Schegget J, Burger MP, Van Der Vange N: Epidemiologic and mucosal immunologic aspects of HPV infection and HPV-related cervical neoplasia in the lower female genital tract: a review. Int J Gynecol Cancer 2001;11:9-17.

12 Howie HL, Katzenellenbogen RA, Galloway DA: Papillomavirus E6 proteins. Virology 2009 20;384:324-334.

13 Wu MH, Chan JY, Liu PY, Liu ST, Huang SM: Human papillomavirus E2 protein associates with nuclear receptors to stimulate nuclear receptor- and E2-dependent transcriptional activations in human cervical carcinoma cells. Int J Biochem Cell Biol 2007;39:413-425.

14 Xu F, Cao M, Shi Q, Chen H, Wang Y, Li X: Integration of the full-length HPV16 genome in cervical cancer and Caski and Siha cell lines and the possible ways of HPV integration. Virus Genes 2015;50:210-220.

15 Schwarz E, Freese UK, Gissmann L, Mayer W, Roggenbuck B, Stremlau A, zur Hausen H: Structure and transcription of human papillomavirus sequences in cervical carcinoma cells. Nature 1985;314:111-114.

16 Smotkin D, Wettstein FO: Transcription of human papillomavirus type 16 early genes in a cervical cancer and a cancer-derived cell line and identification of the E7 protein. Proc Natl Acad Sci U S A 1986;83:46804684 .

17 Smith EM, Pawlita M, Rubenstein LM, Haugen TH, Hamsikova E, Turek LP: Risk factors and survival by HPV-16 E6 and E7 antibody status in human papillomavirus positive head and neck cancer. Int J Cancer 2010;127:111-117.

18 Smith JA, Haberstroh FS, White EA, Livingston DM, DeCaprio JA, Howley PM: SMCX and components of the TIP60 complex contribute to E2 regulation of the HPV E6/E7 promoter. Virology 2014;468-470:311-321.

19 Reuschenbach M, Huebbers CU, Prigge ES, Bermejo JL, Kalteis MS, Preuss SF, Seuthe IM, Kolligs J, Speel EJ, Olthof N, Kremer B, Wagner S, Klussmann JP, Vinokurova S, von Knebel Doeberitz M: Methylation status of HPV16 E2-binding sites classifies subtypes of HPV-associated oropharyngeal cancers. Cancer 2015;121:1966-1976.

20 Cheung JL, Cheung TH, Yu MY, Chan PK: Virological characteristics of cervical cancers carrying pure episomal form of HPV16 genome. Gynecol Oncol 2013;131:374-379. 
21 Anayannis NV, Schlecht NF, Ben-Dayan M, Smith RV, Belbin TJ, Ow TJ, Blakaj DM, Burk RD, Leonard SM, Woodman CB, Parish JL, Prystowsky MB: Association of an intact E2 gene with higher HPV viral load, higher viral oncogene expression, and improved clinical outcome in HPV16 positive head and neck squamous cell carcinoma. PLoS One 2018;13:e0191581.

22 McBride AA: Mechanisms and strategies of papillomavirus replication. Biol Chem 2017;398:919-927.

23 Pinidis P, Tsikouras P, Iatrakis G, Zervoudis S, Koukouli Z, Bothou A, Galazios G, Vladareanu S: Human papilloma virus' life cycle and carcinogenesis. Maedica (Bucur) 2016;11:48-54.

24 Lutgendorf SK, Costanzo ES: Psychoneuroimmunology and health psychology: an integrative model. Brain Behav Immun 2003;17:225-232.

25 Cvitanović H, Milošević M, Bukvić-Bešlić I, Lugović-Mihić L: Determination of psychological stress, serum immune parameters, and cortisol levels in patients with human papilloma virus. Clin Ther 2020;42:783799.

26. heoharides TC, Stewart JM, Taracanova A, Conti P, Zouboulis CC: Neuroendocrinology of the skin. Rev Endocr Metab Disord 2016;17:287-294.

27 Pondeljak N, Lugović-Mihić L: Stress-induced interaction of skin immune cells, hormones, and neurotransmitters. Clin Ther 2020;42:757-770.

28 Lugović-Mihić L, Ljubesić L, Mihić J, Vuković-Cvetković V, Troskot N, Šitum M: Psychoneuroimmunologic aspects of skin diseases. Acta Clin Croat 2013;52:337-342.

29 Buckner SL, Mouser JG, Dankel SJ, Jessee MB, Mattocks KT, Loenneke JP: The General Adaptation Syndrome: Potential misapplications to resistance exercise. J Sci Med Sport 2017;20:1015-1017.

30 Tsigos C, Kyrou I, Kassi E, Chrousos GP: Stress: Endocrine Physiology and pathophysiology. 2020; In: Feingold KR, Anawalt B, Boyce A, et al. (eds): Endotext [Internet]. South Dartmouth (MA): MDText.com, Inc., 2000.

31 Iftikhar A, Islam M, Shepherd S, Jones S, Ellis I: Cancer and stress: does it make a difference to the patient when these two challenges collide? Cancers (Basel) 2021;13:163.

32 McEwen BS: Physiology and neurobiology of stress and adaptation: central role of the brain. Physiol Rev 2007;87:873-904.

33 Caraffa A, Spinas E, Kritas SK, Lessiani G, Ronconi G, Saggini A., Antinolfi P, Pizzicannella J, Toniato E, Theoharides TC, Conti P: Endocrinology of the skin: intradermal neuroimmune network, a new frontier. J Biol Regul Homeost Agents 2016;30:339-343.

34 Fischer A, Ziogas A, Anton-Culver H: Perception matters: Stressful life events increase breast cancer risk. J Psychosom Res 2018;110:46-53.

35 Peters EM: Stressed skin?--a molecular psychosomatic update on stress-causes and effects in dermatologic diseases. J Dtsch Dermatol Ges 2016;14:233-252.

36 Hunter HJ, Momen SE, Kleyn CE: The impact of psychosocial stress on healthy skin. Clin Exp Dermatol 2015;40:540-546.

37 Van Bodegom M, Homberg JR, Henckens MJAG: Modulation of the hypothalamic-pituitary-adrenal axis by early life stress exposure. Front Cell Neurosci 2017;11:87.

38 Dhabhar FS, McEwen B: Chapter 34 - Bi-directional effects of stress on immune function: possible explanations for salubrious as well as harmful effects. In: Ader R (ed): Psychoneuroimmunology (Fourth Edition), Volume II. Oxford, UK, Elsevier Academic Press, 2007, pp 723-760.

39 Cohen S, Janicki-Deverts D, Doyle WJ, Miller GE, Frank E, Rabin BS, Turner RB: Chronic stress, glucocorticoid receptor resistance, inflammation, and disease risk. Proc Natl Acad Sci USA 2012;109:59955999.

40 Stone AA, Bovbjerg DH, Neale JM, Napoli A, Valdimarsdottir H: Development of common cold symptoms following experimental rhinovirus infection is related to prior stressful life events. Behav Med 1992;18:115-120.

41 Koh D, Yong Y, Ng V, Chia SE: Stress, mucosal immunity, upper respiratory tract infections, and sickness absence. J Occup Environ Med 2002;44:987-988.

42 Fang CY, Miller SM, Bovbjerg DH, Bergman C, Edelson MI, Rosenblum NG, Bove BA, Godwin AK, Campbell DE, Douglas SD: Perceived stress is associated with impaired T-cell response to HPV16 in women with cervical dysplasia. Ann Behav Med 2008;35:87-96.

43 Lu D: The role of psychological stress in cervical and prostate carcinogenesis [PhD Thesis]. Stockholm, Sweden, Department of Medical Epidemiology and Biostatistics Karolinska Institutet, 2017. 
Lugović-Mihić et al.: Psychological Stress, HPV Infection and Carcinogenesis

44 Burd EM: Human Papillomavirus and cervical cancer. Clin Microbiol Rev 2003;16:1-17.

45 Lu D, Sundström K, Sparén P, Fall K, Sjölander A, Dillner J, Helm NY, Adami HO, Valdimarsdóttir U, Fang F: Bereavement is associated with an increased risk of HPV Infection and cervical cancer: an epidemiological study in Sweden. Cancer Res 2016;76:643-651.

46 Vonsky M, Shabaeva M, Runov A, Lebedeva N, Chowdhury S, Palefsky JM, Isaguliants M: Carcinogenesis associated with Human Papillomavirus infection. Mechanisms and potential for immunotherapy. Biochemistry (Mosc) 2019;84:782-799.

47 Ziemssen T, Kern S: Psychoneuroimmunology--cross-talk between the immune and nervous systems. J Neurol 2007;254:8-11.

48 Dhabhar FS, Malarkey WB, Neri E, McEwen BS: Stress-induced redistribution of immune cells--from barracks to boulevards to battlefields: a tale of three hormones. Psychoneuroendocrinology 2012;37:13451368.

49 Stefanski V: Social stress affects migration of blood T cells into lymphoid organs. J Neuroimmunology 2003;138:17-24.

50 Goebel MU, Mills PJ: Acute psychological stress and exercise and changes in peripheral leukocyte adhesion molecule expression and density. Psychosom Med 2000;62:664-670.

51 Paik IH, Toh KY, Lee C, Kim JJ, Lee SJ: Psychological stress may induce increased humoral and decreased cellular immunity. Behav Med 2000;26:139-141.

52 Ohta M, Ohmori T, Kawai K, Teshima-Kondo S, Rokutan K: Expression analysis of psychological stressassociated genes in peripheral blood leukociti. Neurosci Lett 2005;381:57-62.

53 Armaiz-Pena GN, Gonzalez-Villasana V, Nagaraja AS, Rodriguez-Aguayo C, Sadaoui NC, Stone RL, Matsuo K, Dalton HJ, Previs RA, Jennings NB, Dorniak P, Hansen JM, Arevalo JM, Cole SW, Lutgendorf SK, Sood AK, Lopez-Berestein G: Adrenergic regulation of monocyte chemotactic protein 1 leads to enhanced macrophage recruitment and ovarian carcinoma growth. Oncotarget 2015;6:4266-73.

54 Jensen MM: The influence of stress on murine leukemia virus infection. Proc Soc Exp Biol Med 1968;127:610-614.

55 Magnon C, Hall SJ, Lin J, Xue X, Gerber L, Freedland SJ, Frenette PS: Autonomic nerve development contributes to prostate cancer progression. Science 2013;341:1236361.

56 Powell ND, Tarr AJ, Sheridan JF: Psychosocial stress and inflammation in cancer. Brain Behav Immun 2013;30:S41-47.

57 Hunzeker J, Padgett DA, Sheridan PA, Dhabhar FS, Sheridan JF: Modulation of natural killer cell activity by restraint stress during an influenza A/PR8 infection in mice. Brain Behav Immun 2004;18:526-535.

58 Thaker PH, Han LY, Kamat AA, Arevalo JM, Takahashi R, Lu C, Jennings NB, Armaiz-Pena G, Bankson JA, Ravoori M, Merritt WM, Lin YG, Mangala LS, Kim TJ, Coleman RL, Landen CN, Li Y, Felix E, Sanguino AM, et al.: Chronic stress promotes tumor growth and angiogenesis in a mouse model of ovarian carcinoma. Nat Med 2006;12:939-944.

59 Verza FA, Valente VB, Oliveira LK, Kayahara GM, Crivelini MM, Furuse C, Biasoli ÉR, Miyahara GI, Oliveira SHP, Bernabé DG: Social isolation stress facilitates chemically induced oral carcinogenesis. PLoS One 2021;16:e0245190.

60 Chang HY, Keyes KM, Mok Y, Jung KJ, Shin YJ, Jee SH: Depression as a risk factor for overall and hormonerelated cancer: the Korean cancer prevention study. J Affect Disord 2015;173:1-8.

61 Chida Y, Hamer M, Wardle J, Steptoe A: Do stress-related psychosocial factors contribute to cancer incidence and survival? Nat Clin Pract Oncol 2008;5:466-475.

62 Coker AL, Bond SM, Pirisi LA: Life stressors are an important reason for women discontinuing follow-up care for cervical neoplasia. Cancer Epidemiol Biomarkers Prev 2006;15:321-329.

63 Cui B, Peng F, Lu J, He B, Su Q, Luo H, Deng Z, Jiang T, Su K, Huang Y, Ud Din Z, Lam EW, Kelley KW, Liu Q: Cancer and stress: NextGen strategies. Brain Behav Immun 2021;93:368-383.

64 DePunzio C, Salvestroni C, Guazzelli G, Papa MC, Freschi G, Ferdeghini M, Masoni S: Stress and cervical dysplasia. Eur J Gynaecol Oncol 1998;19:287-290.

65 Lu D, Andrae B, Valdimarsdóttir U, Sundström K, Fall K, Sparén P, Fang F: Psychological distress is associated with cancer-specific mortality among patients with cervical cancer. Cancer Res 2019;28:canres.0116.2019.

66 Fang F, Fall K, Sparén P, Adami HO, Valdimarsdóttir HB, Lambe M, Valdimarsdóttir U: Risk of infectionrelated cancers after the loss of a child: a follow-up study in Sweden. Cancer Res 2011;71:116-122. 
67 Ikeda A, Kawachi I, Iso H, Iwasaki M, Inoue M, Tsugane S: Social support and cancer incidence and mortality: the JPHC study cohort II. Cancer Causes Control 2013;24:847-860.

68 Kravdal O: The impact of marital status on cancer survival. Soc Sci Med. 2001;52:357-368.

69 Pereira DB, Antoni MH, Danielson A, Simon T, Efantis-Potter J, Carver CS, Durán RE, Ironson G, Klimas N, O’Sullivan MJ: Life stress and cervical squamous intraepithelial lesions in women with human papillomavirus and human immunodeficiency virus. Psychosom Med 2003;65:427-434.

70 Wilkerson JE, Bailey JM, Bieniasz ME, Murray SI, Ruffin MT: Psychosocial factors in risk of cervical intraepithelial lesions. Int J Women Health 2009;18:513-518.

71 Dhabhar FS, Saul AN, Holmes TH, Daugherty C, Neri E, Tillie JM, Kusewitt D, Oberyszyn TM: High anxiety is associated with higher chronic stress burden, lower protective immunity, and increased cancer progression. PLoS One 2012;7:e33069 92.

72 Kołacz R, Grudnik T, Stefaniak T, Bodak E: Haptoglobin in the blood serum of pigs exposed to transportation stress. ISAH, Mexico 2003. URL: http://www.isahsoc.org/userfiles/downloads/ proceedings/2003/speakers/S25KolaczPoland.pdf

73 Van Hunsel F, Van Gastel A, Neels H, Wauters A, Demedts P, Bruyland K, DeMeester I, Scharpé S, Janca A, Song C, Maes M: The influence of psychological stress on total serum protein and patterns obtained in serum protein electrophoresis. Psychol Med 1998;28:301-309.

74 Cole SW, Mendoza SP, Capitanio JP: Social stress desensitizes lymphocytes to regulation by endogenous glucocorticoids: Insights from in vivo cell trafficking dynamics in rhesus macaques. Psychosom Med 2009;71:591-597.

75 Irwin M, Daniels M, Smith TL, Bloom E, Weiner H: Impaired natural killer activity during bereavement. Brain Behav Immun 1987;1:98-104.

76 Sharp L, Cotton S, Carsin AE, Gray N, Thornton A, Cruickshank M, Little J, TOMBOLA Group: Factors associated with psychological distress following colposcopy among women with low-grade abnormal cervical cytology: a prospective study within the Trial of management of borderline and other low-grade abnormal smears (TOMBOLA). Psychooncology 2013;22:368-380.

77 Bond E, Lu D, Herweijer E, Sundström K, Valdimarsdóttir U, Fall K, Arnheim-Dahlström L, Sparén P, Fang F: Sexually transmitted infections after bereavement - a population-based cohort study. BMC Infect Dis 2016;16:419.

78 Kola S, Walsh JC, Hughes BM, Howard S: Matching intra-procedural information with coping style reduces psychophysiological arousal in women undergoing colposcopy. J Behav Med 2013;36:401-412.

79 Massad LS, Agniel D, Minkoff H, Watts DH, D'Souza G, Levine AM: Effect of stress and depression on the frequency of squamous intraepithelial lesions. J Low Genit Tract Dis 2011;15:42-47.

80 Antoni MH, Pereira DB, Marion I, Ennis N, Andrasik MP, Rose R, McCalla J, Simon T, Fletcher MA, Lucci J, Efantis-Potter J, O'Sullivan MJ: Stress management effects on perceived stress and cervical neoplasia in low-income HIV-infected women. J Psychosom Res 2008;65:389-401.

81 Giuliano AR, Nyitray AG, Kreimer AR, Pierce Campbell CM, Goodman MT, Sudenga SL, Monsonego J, Franceschi S: EUROGIN 2014 roadmap: differences in human papillomavirus infection natural history, transmission and human papillomavirus-related cancer incidence by gender and anatomic site of infection. Int J Cancer 2015;136:2752-2760.

82 McCaffery K, Waller J, Forrest S, Cadman L, Szarewski A, Wardle J: Testing positive for human papillomavirus in routine cervical screening: examination of psychosocial impact. BJOG 2004;111:14371443.

83 Tomás-Aragonés L, Castillo-Amores AB, Rodríguez-Cerdeira C, Marrón-Moya SE: Psychological aspects associated with the acquisition and development of HPV infection and its repercussion on quality of life. Open Dermatol J 2009;3:133-136.

84 Jensen SE, Pereira DB, Whitehead N, Buscher I, McCalla J, Andrasik M, Rose R, Antoni MH: Cognitivebehavioral stress management and psychological well-being in HIV+ racial/ethnic minority women with human papillomavirus. Health Psychol 2013;32:227-230.

85 Jentschke M, Lehmann R, Drews N, Hansel A, Schmitz M, Hillemanns P: Psychological distress in cervical cancer screening: results from a German online survey. Arch Gynecol Obstet 2020;302:699-705. 